|| Print ISSN: 2589-7837 || Online ISSN: 2581-3935 ||

International Journal of Medical Science and Diagnosis Research (IJMSDR)

Available Online at www.ijmsdr.com

NLM (National Library of Medicine ID: 101738824)

\title{
Pink Tooth in Forensic Dentistry- A Short Review
}

\author{
Dr. Shaik Mohamed Shamsudeen ${ }^{1}$ \\ ${ }^{1}$ Assistant professor, Department of Dental Diagnostic science, College of Dentistry, King \\ Khalid University, Abha, KSA
}

\section{Conflicts of Interest: Nil}

Corresponding author: Dr. Shaik Mohamed Shamsudeen

DOI: https://doi.org/10.32553/ijmsdr.v6i1.903

\begin{abstract}
:
A pink tooth is common phenomenon that can be noted by the forensic personnel during the postmortem and evaluations of their causes in forensic dentistry following death are important aspects. It was considered a cadaveric alteration expressed after violent deaths. More recently, studies explain that the phenomenon may manifest induced by the environment factors. Forensic experts and dentists must be aware of the occurrence of the pink tooth phenomenon. This article reviews the causes, pathogenesis and histological aspects of the pink teeth phenomenon in the forensics dentistry.

Keywords: Pink tooth, Forensic dentistry, Postmortem, Haemolysis.
\end{abstract}

\section{Introduction:}

Pink tooth in dentistry is commonly referred as a synonym for internal tooth resorption. The occurrence of pinkish discoloration of the teeth can be localized or generalized. Their cause is intrinsic or extrinsic related with trauma leading to pulpal necrosis. ${ }^{1}$ In forensic odontology, pink tooth was considered as an important finding in postmortem examination. The first reported case of pink tooth in the literature was by Bell in which the cause of the death was drowning. ${ }^{2}$.Later many cases were reported by forensic personnel regarding pink tooth and Christie case gained attention in which pink tooth was an important evidence in forensic field. ${ }^{3}$

\section{Causes}

The common cause of death in pink tooth was related to asphyxia such as strangulation, drowning and suffocation. ${ }^{4}$ It was observed in the literature that pink tooth is often seen in drowning conditions where the head is in downward position than the trunk. The humid environmental condition and advanced decomposition favors the development of the pink tooth. ${ }^{5}$ The sudden death is also one of the important factor in developing pink teeth as the blood will remain in liquid state, due coagulation process is delayed. In a study it showed that the fibrinolytic activity and catecholamine are higher in sudden death maintaining the blood in the liquid state. In strangulation, the hemoglobin forms an unstable complex with carbon monoxide which causes pink tooth to form. ${ }^{6}$ In a case series, three out of eight cases, the body was recovered from water showing pink teeth. ${ }^{7}$

The time of development of pink tooth may vary from weeks to years. They do not occur immediately after death, and most cases reported after a week as the hemolysis develop and peak during this period. Van Wyk experiment in vitro showed pink tooth developed after 6 days and the color was peak 
at 18th day. The color of the tooth will fade away after a year of its occurrence. ${ }^{8}$

Postmortem pink tooth is common in young age groups due to larger dentinal tubules and diffusion of hemoglobin into the dentinal tubules is easier. Because of the sclerosed dentin, it was rarely seen in the older age. Anterior teeth are affected commonly than the posterior teeth. Cementoenamel junction is the most commonly affected area on the tooth. The root shows more intense discoloration than the crown. ${ }^{9}$

\section{Pathogenesis}

The development of pinkish appearance of the teeth was considered due to entering of red blood cells into the dentinal tubule. But the red blood cells cannot enter directly into the dentinal tubules as it is bigger than tubule. The increased pressure of the blood vessels in the pulp causes lysis of erythrocytes and the products of erythrocytes like hemoglobin, porphyrins, and hemosiderin, together with bile and related pigments are entering the dentinal tubules makes the tooth pink in color. $^{10}$

\section{Histopathology:}

The ground sectioned of the pink tooth showed red-brown discoloration of the dentin. Enamel and cementum was normal without any changes. The decalcified hematoxylin and eosin stained section does not show any notable changes. ${ }^{11}$

\section{Conclusion:}

Pink teeth in forensic dentistry have been noted in sudden death whereas moist environment plays a triggering role. The exact cause is not still known, the diffusion of red cell products into the dentinal tubules are responsible for their appearance. Hence further studies should be carried out in research field to identify its importance in forensic field.

\section{References}

1. Beeley JA, Harvey W. Pink teeth as a postmortem phenomenon. J Forensic SciSoc 1973;13:297-305.

2. Bell T. Anatomy, physiology and disease of the teeth. Highley, London (1829): 1213.

3. Camps FE. Medical and scientific investigations in the Christie case (1953). Achar Press, Tiptree Esser.

4. Borrman H, Du Chesne A, Brinkmann B. Medico-legal aspects of postmortem pink teeth. Int J Legal Med. 1994;106:225-31

5. Inoue $T$, Hatake K. Forensic Significance of the Post-mortem Pink Teeth. Res Pract Forensic Med. 2001;44:291-8.

6. Van Wyk CW. Postmortem pink teeth. Histochemical identification of the causative pigment Am J Forens Med Pathol1989; 10:134-139.

7. AdemirFrancoa, Solon Diego Santos Carvalho Mendes, Fernando Fortes Picoli , Livia Graziele Rodrigues, Rhonan Ferreira Silva. Forensic thanatology and the pink tooth phenomenon: From the lack of relation with the cause of death to a potential evidence of cadaveric decomposition in dental autopsies - Case series. Forensic Science InternationalVolume , October 2018;291: e8-e12.

8. Van Wyk CW. Pink teeth of the dead: 1. A clinical and histological description. J Forensic Odontostomatol. 1987;5:41-50.

9. RaveenaThapar, Swati Choudhry, Anju Sinha, Ruchita Bali, Deepika Shukla. Pink tooth phenomenon: an enigma?J Forensic Leg Med 2013 Oct;20(7):912-4.

10. Evelyne-Pessoa Soriano , Marcus-VitorDiniz de Carvalho, Francisco-Bernardo Dos Santos , Clóvis-César de Mendoza , Maria-do Socorro-Dantas de Araújo, Reginaldo-Inojosa-CarneiroCampello . The post-mortem pink teeth phenomenon: A case report. Med Oral Patol Oral Cir Bucal. 2009 Jul 1;14 (7):E337-9. 
11. BK Charan Gowda, B appearance of postmortem pink teeth: Sivapathasundharam, Ananjan Report of two cases. J Forensic Dent Sci. Chatterji, and BL Chatterji. Histological 2015; 7(3): 260 